\title{
The status of KRAS mutations in patients with non-small cell lung cancers from mainland China
}

\author{
MEI LI ${ }^{1}$, LINA LIU ${ }^{3}$, ZHIMIN LIU ${ }^{1}$, SHICHANG YUE ${ }^{2}$, LUMING ZHOU $^{5}$, QIUPING ZHANG ${ }^{4}$, \\ SHIHUA CHENG ${ }^{4}$, RACHEL W. $\mathrm{LI}^{6,7}$, PAUL N. SMITH ${ }^{7}$ and SHEN LU ${ }^{1}$
}

\author{
${ }^{1}$ Laboratory Center, and ${ }^{2}$ Department of Thoracic Surgery, The Second Affiliated Hospital of Dalian Medical University; \\ Departments of ${ }^{3}$ Gastroenterology and ${ }^{4}$ Pathology, The First Affiliated Hospital of Dalian Medical University, Dalian 116027, \\ Liaoning Province, P.R. China; ${ }^{5}$ Department of Pathology, University of Utah School of Medicine, Salt Lake City, \\ UT 84132, USA; ${ }^{6}$ Department of Surgery, Medical School, The Australian National University, Canberra, \\ ACT 2601; ${ }^{7}$ Department of Surgery, The Canberra Hospital, Canberra, ACT 2606, Australia
}

Received July 6, 2009; Accepted August 10, 2009

DOI: 10.3892/or_00000529

\begin{abstract}
KRAS mutation is closely associated to carcinogenesis and prognosis of non-small cell lung cancer (NSCLC). Detection of KRAS mutation can also be used to select NSCLC patients for drug targeting with EGFR tyrosine kinase inhibitors. Data regarding the status of KRAS mutation in mainland China, which would assist in these interventions, is lacking. We have detected KRAS mutation from 103 NSCLC patients in mainland China with high resolution melting analysis (HRM) on LightScanner ${ }^{\mathrm{TM}}$, and compared this method of detection with sequencing, and found HRM to have greater sensitivity. We found 6 patients $(5.8 \%)$ with KRAS mutation ( 3 patients, G12C; 1 patient, G12S; 1 patient, G12V; 1 patient, G13D). KRAS mutation was significantly associated to gender $(\mathrm{p}=0.027)$ and pathology types $(\mathrm{p}=0.000)$, but not to smoking. The mutation frequency of KRAS in NSCLC patients in mainland China is similar to those in East Asian countries, but lower than those in Western countries. However, the spectrum of KRAS mutation in mainland China is similar to those found in the USA. The results also exhibit dependence of KRAS mutation in China on ethnicity. The clinical significance of the spectral pattern of KRAS mutations in TKI resistance or tumorigenesis among patients with NSCLC in mainland China requires further investigation.
\end{abstract}

Correspondence to: Dr Shen Lu, Laboratory Center, The Second Affiliated Hospital of Dalian Medical University, No. 467, Zhongshan Road, Shahekou District, Dalian 116027, Liaoning Province, P.R. China

E-mail: 1vshen1956@yahoo.com.cn

Key words: KRAS, mutation, non-small cell lung cancer, high resolution melting

\section{Introduction}

Lung cancer is one of the most frequent and deadly malignant tumors. Non-small cell lung cancers (NSCLCs) comprise about $80 \%$ of lung cancers (1). Its carcinogenesis is still not clear. Abnormal activation of the RAS/MAPK signal transduction pathway, that plays a key role in the regulatory processes of proliferation, differentiation, apoptosis and angiogenesis in cells, is closely associated with NSCLC carcinogenesis $(2,3)$. Kirsten RAS (KRAS) is an important member of the RAS oncogene family and a pivotal signal molecule in the pathway (4). Epithelial growth factor receptor (EGFR) gene mutations can activate the RAS/MAPK pathway abnormally by activating KRAS and then lead to NSCLC carcinogenesis $(5,6)$. Therefore, EGFR tyrosine kinase inhibitor (TKI) target drugs such as Gefitinib and Erlotinib, show a good therapeutic response to NSCLC patients with EGFR mutations by inhibiting EGFR from activating the downstream signal of the RAS pathway (7-9). However, NSCLC patients with KRAS mutations usually have bad therapeutic responses to EGFR TKIs, since KRAS mutations themselves, which are mostly clustered on codon 12 and 13, can also activate the RAS/MAPK pathway abnormally and then lead to NSCLC carcinogenesis $(4,10-13)$. In addition, because KRAS mutations are often mutually exclusive to EGFR mutations, KRAS mutations may be used as a helpful complementary biomarker to select sensitive patients with NSCLC to EGFR TKIs (11-13). Interestingly, EGFR mutation frequency is high in the patients with NSCLC from East Asia, while low in patients from Western countries (14). However, the variation in mutation frequency between these populations (among patients with NSCLC) for KRAS is contrary to that of EGFR. It was reported that KRAS mutation frequency is about $30 \%$ or more in NSCLC patients from the USA and some other Western countries, while it was less than $10 \%$ in NSCLC patients from Japan, Korea, Taiwan and Hong Kong in China $(1,15-22)$. It is indicated that EGFR and KRAS gene mutations in NSCLC patients may both be dependent on the ethnicity, but the ordering of the mutation frequencies is 
reversed. The study is to supplement the data from mainland China.

The relationship between KRAS mutations and prognosis is also discussed in the literature, showing that KRAS mutations are often related to a poor prognosis in some lung cancer patients but not others. Later it was found that the prognostic value was dependent on the specific spectrum of the KRAS mutation (14-17,23). The KRAS mutation spectra in lung cancer patients from America and Europe are different, as are those of African-American and Caucasian patients (24). These studies indicate that the spectrum of KRAS mutations may be different among different ethnicities. This suggests that it would be useful to study the spectra of KRAS mutation in NSCLC patients from mainland China.

High resolution melting (HRM) is a sensitive, rapid and low cost technique for mutation scanning. This technique has been widely used in clinical diagnostics and research (25-27). In this study, we used the method of high resolution melting analysis to detect KRAS mutations in NSCLC patients in mainland China. Our results provide a first report of KRAS mutations in NSCLC in the mainland Chinese population.

\section{Materials and methods}

Patients and tissue samples. Tumor specimens, along with matched normal lung tissues around tumor tissue were collected from 103 patients with NSCLC who underwent surgical procedures at the affiliated hospitals of the Dalian Medical University from July 2007 to August 2008. Of the 103 patients, 54 were men and 59 were women with a mean \pm SD age of $62.2 \pm 9.3$ years (range $38-80$ years). All tumors were diagnosed by two certified pathologists. The tumors included 32 squamous cell carcinomas (SCC), 69 adenocarcinomas (Adc) and 2 large cell carcinomas (LCC). Of the 69 adenocarcinomas, there were 16 mixed adenocarcinomas with bronchioloalveolar components (AWBC) or bronchioloalveolar adenocarcinomas (BAC). Clinical data of the patients' smoking history, clinical stage and lymph node metastasis are presented in Table II. All tissue samples were collected with the approval of The Ethics Committee of the Dalian Medical University and with the informed consent of the patients or families before surgical procedures.

Cell lines. Four human cell lines, A549, HCT116, RPMI8226 and K562 were sourced from the Cell Culture Center of the Institute of Basic Medical Sciences, Chinese Academy of Medical Sciences School. The A549 cell line contains a homozygous KRAS mutation (G12S), the HCT116 cell line contains a heterozygous KRAS mutation (G13D), the RPMI8226 cell line contains a heterozygous KRAS mutation (G12A) (10), and the K562 cell line contains the wild-type of KRAS (Table I). A549 cells were grown in F12 (Hyclone, Thermo Fisher Scientific Inc.), HCT116 cells were grown in DMEM (Gibco, Invitrogen), RPMI8226 and K562 cells were grown in RPMI-1640 (Gibco, Invitrogen). All cell lines were cultured as instructed by the supplier.

DNA extraction. Every sample, either tumor or the matched normal lung tissue, was prepared as a frozen section, and stained with hematoxylin and eosin. Two pathologists confirmed the tumor-rich areas and normal lung tissue areas, and then marked the selected area on the stained slides. Genomic DNA were extracted from the tumor tissues, the matched normal lung tissues and cell lines A549, HCT116, RPMI8226 and K562 using a Cell/Tissue Genomic DNA Purification Kit (Tiangen-Qiagen, Beijing, China) according to the manufacturer's protocol.

PCR condition and high resolution melting analysis. PCR was performed in a total volume of $10 \mu 1$ reaction mixture with a $15 \mu 1$ mineral oil overlay in each well on a 96-well PCR plate. The reaction mixture contained 1X PCR buffer with $1.5 \mathrm{mM} \mathrm{MgCl}_{2}$ (Takara), $200 \mu \mathrm{M}$ dNTPs, $0.25 \mathrm{U}$ Taq polymerase (Takara), $20 \mathrm{ng}$ genomic DNA, $0.5 \mu \mathrm{M}$ forward primer, $0.5 \mu \mathrm{M}$ reverse primer, and 1X LCGreen PLUS ${ }^{\circledR}$ (Idaho Technology). Primers were designed to span codons 12 and 13 of KRAS gene exon 2 with a 167 base pair (bp) amplicon. The primer sequences were 5'-AGGCCTGCTGA AAATGACT-3' (forward) and 5'-AATGGTCCTGCACCA GTAA-3' (reverse). PCR was performed on a Mastercycler (Eppendorf) with an initial denaturation for $2 \mathrm{~min}$ at $95^{\circ} \mathrm{C}$, followed by 45 cycles consisting of a denaturation step at $95^{\circ} \mathrm{C}$ for $30 \mathrm{sec}$, an annealing step at $61^{\circ} \mathrm{C}$ for $10 \mathrm{sec}$, and an extension step at $72^{\circ} \mathrm{C}$ for $30 \mathrm{sec}$.

In a 96-well PCR plate, each sample was analyzed in triplicate. Deionized water was used in a no-template control well (no genomic DNA was added) and DNA K562 was used as a wild-type control sample. The tumor tissue and the matched normal lung tissue from the same patients were analyzed in the same PCR plate. When samples were amplified in 96-well plates, melting curve acquisition was performed on the LightScanner (Idaho Technology). The PCR products and melting curves were analyzed using the LightScanner software call-it (version 1.5) according to the manufacturer's instructions.

DNA sequencing. In order to compare the sensitivity of HRM with that of sequencing as described below, some PCR products were sequenced with a BigDye Terminator v3.1 Cycle Sequencing Kit, performed on an ABI PRISM 3730XL DNA Analyzer and an ABI PRISM 377XL DNA Sequencer.

Statistics. Associations between the types of KRAS mutation detected and clinical pathological and histological features were evaluated with Fisher's exact test and non-parametric test using SPSS 13.0 statistical software. A p-value of $<0.05$ was considered significant.

\section{Results}

The 96-well LightScanner sensitivity study. Four cell lines were used to determine the sensitivity of the 96-well LightScanner to the allele fraction of the various mutations of interest. DNA with mutations in KRAS from cell lines RPMI8226 (heterozygous $35 \mathrm{G}>\mathrm{C}$ ), HCT116 (heterozygous $34 \mathrm{G}>\mathrm{A}$ ), A549 (homozygous $35 \mathrm{G}>\mathrm{A}$ ) was separately mixed with wild-type K562 DNA in proportions of 2, 5, 10, 20, 30, $40,50,60,70,80,90$ and $100 \%$. Mutation detection was performed separately by high resolution melting analysis on 
Table I. Cell lines with mutated KRAS and wild-type KRAS.

\begin{tabular}{lllll}
\hline Cell line & \multicolumn{1}{c}{ Tissue } & Mutation & Genotype & Amino acid \\
\hline RPMI8226 & Plasma cell myeloma & $35 \mathrm{G}>\mathrm{C}$ & Heterozygous & G12A \\
HCT116 & Colon carcinoma & $38 \mathrm{G}>\mathrm{A}$ & Heterozygous & G13D \\
A549 & Lung carcinoma & $34 \mathrm{G}>\mathrm{A}$ & Homozygous & G12S \\
K562 & Chronic myelogenous leukemia & Wild-type & & \\
\hline
\end{tabular}
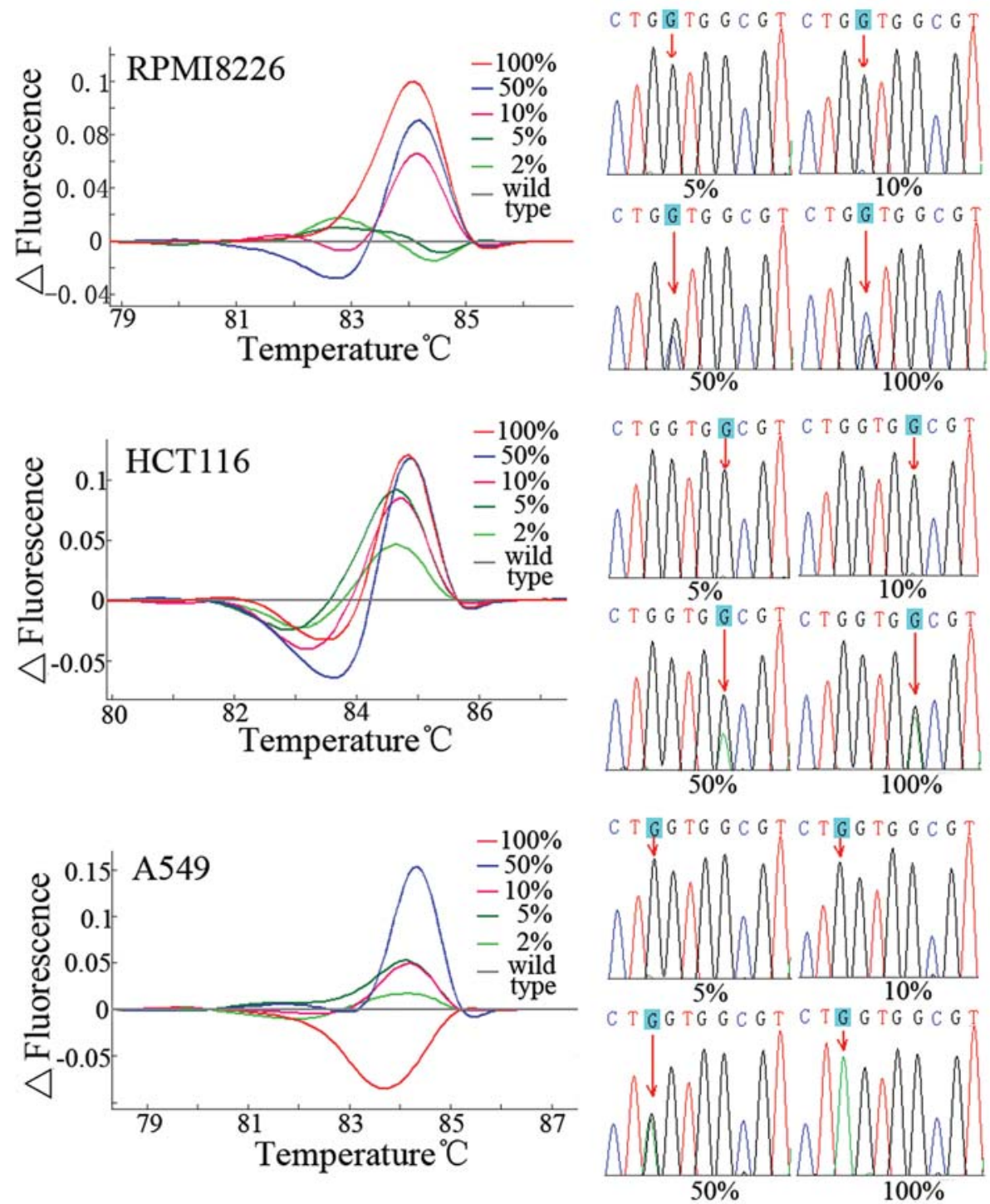

Figure 1. The plot trend of 3 kinds of KRAS mutation (RPMI8226, HCT116, A549) in serial dilutions ranging from 2, 5, 10 to $100 \%$ to the wild-type KRAS (K562) detected with HRM (left) and DNA sequencing (right).

the 96-well LightScanner and by DNA sequencing. Fig. 1 shows the different melting plots of 3 mutations with wildtype K562 DNA in a range of dilutions 2, 5, 10, 50 and $100 \%$. In the 2 and $5 \%$ proportions of RPMI8226 with wildtype K562 DNA, there was no obvious difference between the melting curves of DNA containing mutations and wildtype DNA. However, from 10 to $100 \%$, the melting curves could be readily distinguished. For HCT116 and A549, the melting curves of proportion $5 \%$ and above were distinguished from the wild-type baseline. Each dilution of the three cell lines was also analyzed by sequencing. In mutation fractions of $<20 \%$, mutations were below the threshold of detection by sequencing. In contrast, HRM could sometimes detect mutation allele fractions as low as $2.5 \%$. 

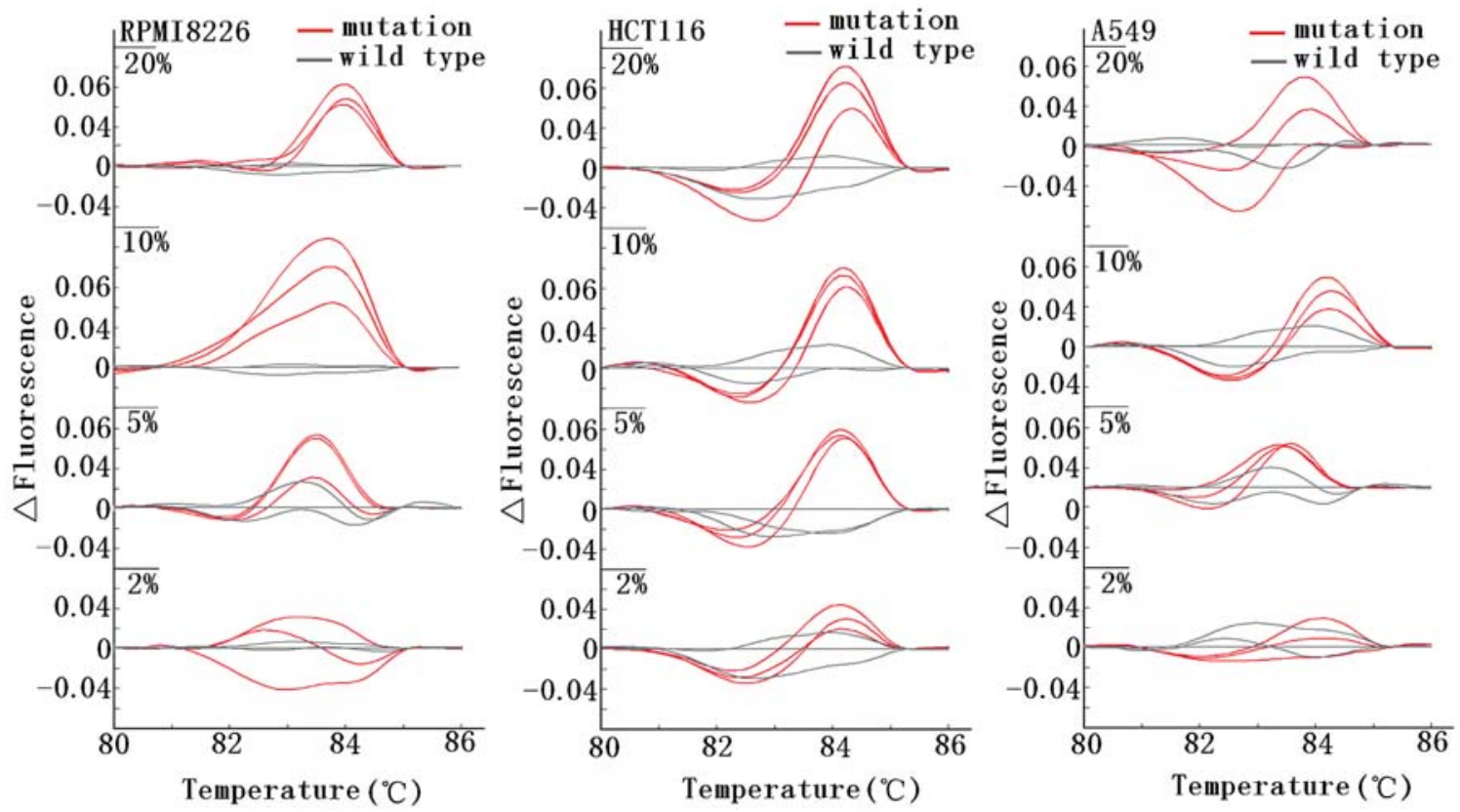

Figure 2. Different proportions of KRAS mutation (RPMI8226, HCT116 and A549: 2, 5, 10, 20\%) detected with HRM on LightScanner. Each proportion of KRAS mutation DNA was compared to wild-type KRAS and each sample was analyzed in triplicate.

Table II. Clinicopathological characters of NSCLC patients with KRAS mutation.

\begin{tabular}{lcclccc}
\hline Case no. & Age & Gender & Smoking history & Pathology & LN metastasis & Mutation spectrum \\
\hline 40 & 40 & Male & Never smoked & AWBC & No & G12C \\
44 & 62 & Male & Smoker & AWBC & Yes & G12S \\
49 & 67 & Male & Never smoked & Adc & No & G13D \\
58 & 62 & Male & Smoker & Adc & Yes & G12C \\
64 & 63 & Male & Smoker & SCC & No & G12C \\
79 & 77 & Male & Never smoked & Adc & No & G12V \\
\hline
\end{tabular}

Adc, adenocarcinoma; AWBC, mixed adenocarcinomas with bronchioloalveolar components; SCC, squamous cell carcinoma.

To enhance the power of the sensitivity study, we repeated the analysis based on triplicate data. Proportions of 2, 5, 10, 20\% mutations RPMI8226, HCT116 and A549 DNA were tested to confirm the sensitivity of mutation detection on a LightScanner (Fig. 2). Because of RPMI8226 is heterozygous mutation KRAS $35 \mathrm{G}>\mathrm{C}$, the distinguishable allele fraction of this mutation is $5 \%$. The distinguishable allele fraction of HCT116 (Heterozygous $34 \mathrm{G}>\mathrm{C}$ ) is $2.5 \%$ and $\mathrm{A} 549$ (homozygous $35 \mathrm{G}>\mathrm{C}$ ) is $5 \%$.

KRAS mutation detection. The NSCLC tissue specimens and their matched normal lung tissues were analyzed for KRAS mutation in exon 2 using HRM on the LightScanner. The tumor sample and normal lung tissue collected from the same subject were analyzed in triplicate and on the same 96-well plate. The samples were detected with the instrument sensitivity setting at normal. Comparing the melting curves of PCR products of all samples, 6 tumor specimens (out of a total of 103) showed KRAS mutation in exon 2 (Fig. 3A and $\mathrm{B}$ ). None of the matched normal lung tissues, (including the 6 cases with KRAS mutation in their tumor samples) showed any KRAS mutations. Their melting curves looked identical to the melting curves of wild-type DNA.

We further sequenced the 6 samples with positive KRAS mutation and 30 samples with negative KRAS mutation randomly selected from tissues analyzed in this study. No false positive cases were detected by HRM (Fig. 3C). Two mutation sites were observed in this study. Codon 12 of KRAS exon 2 exhibited three different mutations ( 3 patients, G12C; 1 patient, G12S; 1 patient, G12V) and one KRAS mutation occurred in codon 13 of KRAS exon 2 (1 patient, G13D).

Correlation of KRAS mutations with clinicopathological characteristics. A total of 103 cases diagnosed as NSCLCs at the two affiliated hospitals of Dalian Medical University. 
A

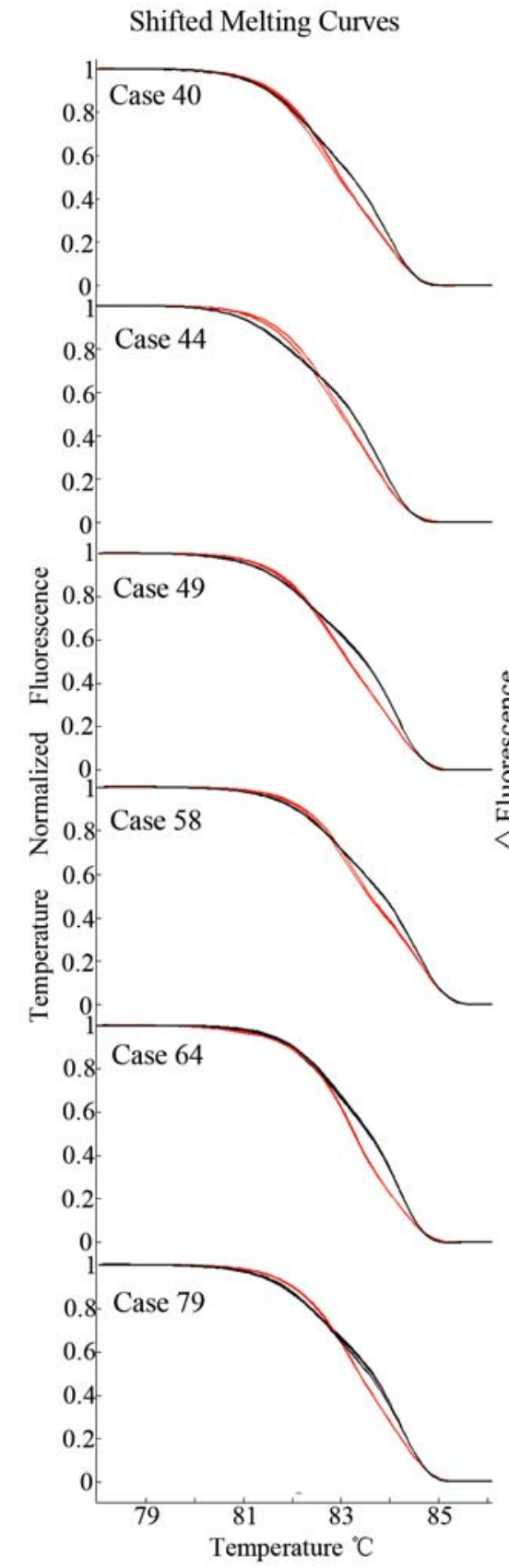

B

Difference Curves

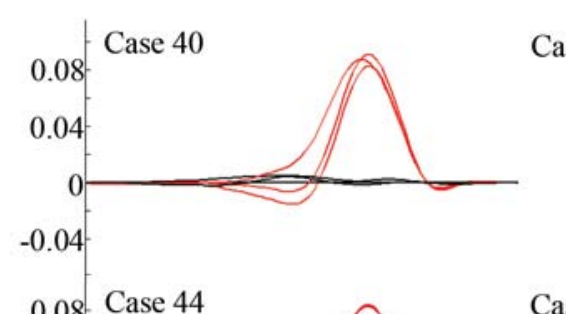

C

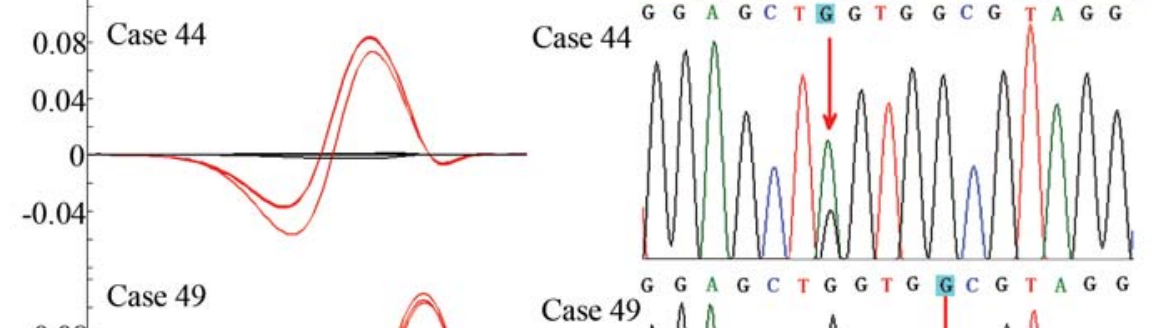

Sequencing
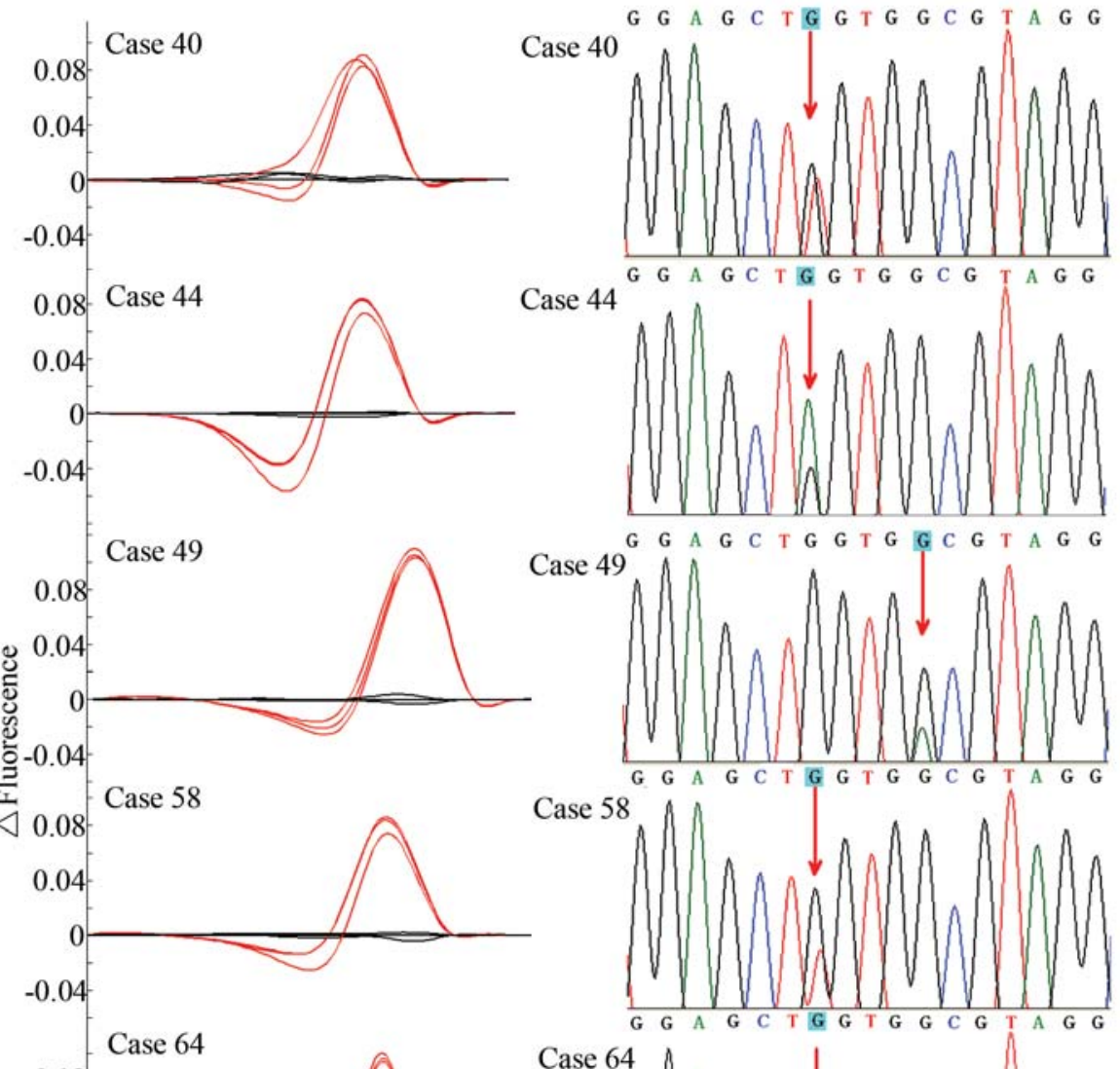

Case 58

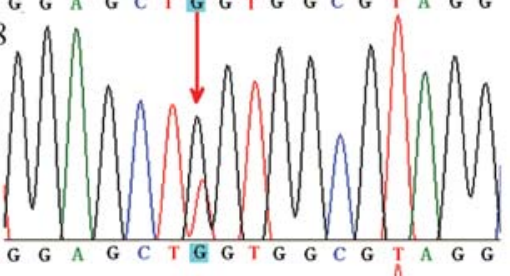

Case 64
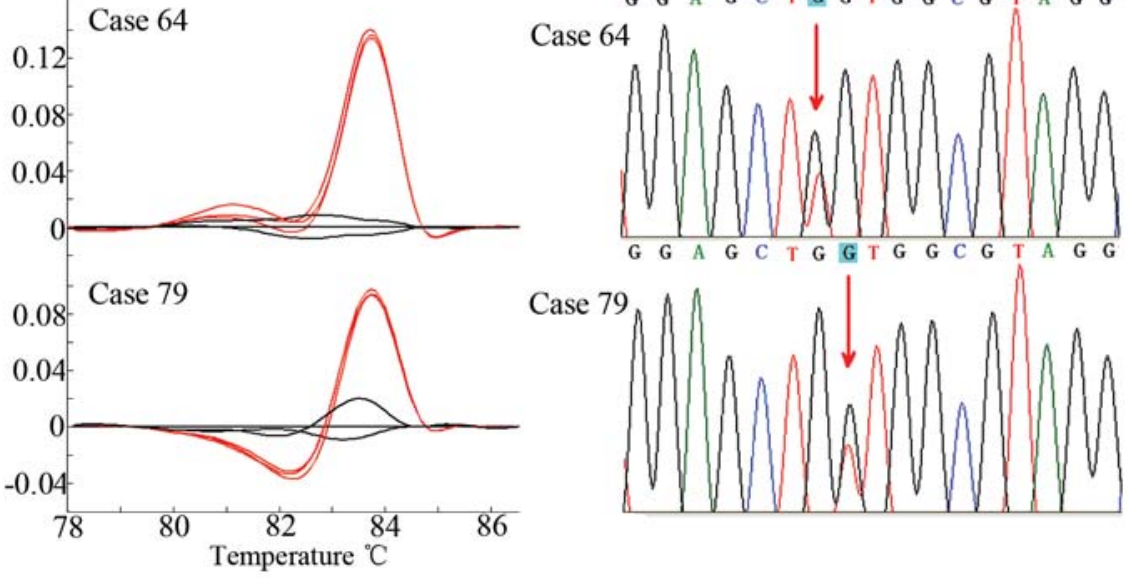

— Tumor tissue — Normal Lung Tissue

Figure 3. The tumor samples with KRAS mutation were compared with matched normal lung tissue from the 6 cases with NSCLC.

The KRAS mutations were found in 6 of 103 cases $(5.8 \%)$. Five of $6(83.3 \%)$ were located in codon 12, and one (16.7\%) was located in codon 13. The substitution of cysteine for glycine in codon 12 comprised $50 \%(3 / 6)$ of all the mutation samples. In the 3 cysteine substitutions, there were 2 patients without lymph node (LN) metastasis and 2 patients had a history of smoking, and in other substitutions, 2 patients without LN metastasis and 1 patient had a smoking history. All 6 patients with KRAS mutation were male with a mean \pm SD age of $61.8 \pm 12.1$ years. As shown in Table III, there were 5 Adc including 2 AWBC and 1 SCC. Our study showed that this KRAS mutation was significantly associated to gender $(\mathrm{p}=0.027)$ and pathology types $(\mathrm{p}=0.000)$, but not to age, smoking history or metastasis (Table III).

\section{Discussion}

Carcinogenesis is a multistep process involving many oncogene activations and tumor suppressor gene inactivations (28). KRAS oncogene is one of the most common mutated oncogenes relating to carcinogenesis. Since it was first found in human lung cancer cells, there have been many reports relating KRAS mutation and lung cancer $(29,30)$. Most of these reports have been from Western countries, but few 
Table III. Correlation between KRAS mutation and clinicopathological characters.

\begin{tabular}{|c|c|c|c|}
\hline Variable & No. of cases & No. $(\%)$ of KRAS mutation & P-value \\
\hline Age & & & 0.233 \\
\hline$>60$ & 57 & $5(8.8)$ & \\
\hline$\leq 60$ & 46 & $1(2.2)$ & \\
\hline Gender & & & 0.032 \\
\hline Male & 54 & $6(1.1)$ & \\
\hline Female & 49 & $0(0)$ & \\
\hline Smoking history & & & 0.664 \\
\hline Non-smokers & 67 & $3(4.5)$ & \\
\hline Smokers & 36 & $3(8.3)$ & \\
\hline Pathology ${ }^{a}$ & & & $0.000^{\mathrm{a}}$ \\
\hline $\mathrm{Adc}^{\mathrm{b}}$ & 69 & $5(7.2)$ & $0.078^{\mathrm{b}}$ \\
\hline $\mathrm{AWBC}$ or $\mathrm{Bac}$ & 16 & $3(18.8)$ & \\
\hline Other Adc & 53 & $2(3.8)$ & \\
\hline SCC & 32 & $1(3.1)$ & \\
\hline $\mathrm{LCC}$ & 2 & $0(0)$ & \\
\hline LN metastasis & & & 0.679 \\
\hline Yes & 51 & $2(3.8)$ & \\
\hline No & 52 & $4(7.1)$ & \\
\hline
\end{tabular}

a Comparison of three pathology types (Adc, SCC and LCC); ${ }^{b}$ comparison of two types of Adc (AWBC or Bac to other Adc).

from East Asia. With the arrival of the molecular target drug EGFR-TKI, the value of KRAS was recognized again as an important signal molecule of EGFR-RAS/MAPK signal transduction pathway. EGFR mutations were common in females, never-smokers, adenocarcinoma patients with NSCLC from East Asia, while not in patients from Western countries $(7,8,31-34)$. KRAS mutation frequency was lower in East Asia than in Western countries. We report here that the frequency of KRAS mutation in 103 NSCLC patients from mainland China is $5.8 \%(6 / 103)$. This result is similar to other countries in Eastern Asia, such as Japan and Korea, where the frequency of KRAS mutation has been reported as less than $10 \%(19,20)$. Moreover, the 6 patients with KRAS mutations were all male, which implies that EGFR mutation may be more closely associated than KRAS mutation with the carcinogenesis of NSCLC in East-Asian countries. It was reported that KRAS mutations were related to smoking history in some Western countries $(35,36)$. We did not observe such a correlation in our research. Consequently, the disorder of EGFR-KRAS/MAPK signal pathway may play a pivotal role in carcinogenesis of NSCLC, but which gene was activated or inactivated may be different depending on different ethnicities.

We found a difference in the patterns of KRAS mutation spectra when comparing our results with the literature reporting from other East Asian countries and areas, although there was a similarity in the frequency of KRAS mutation in these ares $(19,20)$. Furthermore, we compared our results of KRAS mutation spectra to the results reported by Sakuma et al from Japan, Bae et al from Korea, Wu et al from Taiwan, China, Tam et al from Hong Kong in China, all in East Asia, as well as Siegfried et al from the USA, Rodenhuis et al from the Netherlands, and Rosell et al from Spain (15-22). We detected one sample with KRAS mutation in codon 13, and one sample with KRAS mutation in codon 12 with wild-type glycine (GGT) changing to serine. These two rare mutations were not found in the 141 NSCLCs from Japan, the 115 NSCLCs from Korea or the 237 NSCLCs from Taiwan. The spectra of KRAS mutation in codon 12 reported by Tam et al from Hong Kong was exactly the same as that we found, but they did not find the mutation in codon 13. Interestingly, these two mutations were found in the USA, and especially in African-Americans. A G>T transversion in codon 12 (GGT>TGT) resulting in the substitution of cysteine for glycine, was the most common mutation in mainland China (50\%) and Hong Kong (42.86\%) and in Japan $(60 \%)$, but not in Taiwan (0) nor in Korea $(16.7 \%)$. This mutation was also the most common in USA $(44.3 \%)$ and the Netherlands $(55.6 \%)$. The KRAS mutation spectrum may be related to race, but if there are regional distributions in the genetic characters of NSCLC patients then this deserves further investigation.

Detection of KRAS mutations has an important clinical significance in the early diagnosis, treatment and prognosis of NSCLC patients. The method of detection for KRAS mutations must be able to fulfill some criteria, such as high sensitivity, high specificity, repeatability, simplicity and speed. HRM is a new technique with several advantages over 
traditional mutation detection techniques, and which is suited to clinical use for mutation detection with speedy results $(25,26,37,38)$. We compared the corresponding KRAS amplicons obtained from cancer tissue with those from surrounding non-cancer tissue from the same patient in order to avoid variation due to any other polymorphism between different cases. Three cell lines with known KRAS mutation were used to test the sensitivity of the LightScanner and calibrate the appropriate threshold for our detection protocol all in order to minimize false positive and false negative results. In the 6 mutation samples detected with the LightScanner, the randomly selected 30 non-mutation samples were all confirmed by sequencing. Our data provide supporting evidence for the use of HRM on the Lightscanner to clinically detect an identical gene mutation across several cases simultaneously.

In summary, the mutation frequency of KRAS exon 2 in NSCLC patients in mainland China is similar to those in East Asian countries, but lower than those in Western countries. However, the spectrum of KRAS mutations in mainland China is similar to those found in the USA. The clinical significance of the spectral pattern of KRAS mutations in TKI resistance or tumorigenesis among patients with NSCLC in mainland China requires further investigation.

\section{Acknowledgments}

This work was supported by Dalian Merricom gene diagnosis technology Co., Ltd. We thank Dr Robert Palais and Gudrun Reed for critical reading of the manuscript.

\section{References}

1. Parkin M, Tyczynski JE, Boffetta P, Samet J, Shields P and Caporaso N: Lung cancer epidemiology and etiology. In: Pathology and Genetics, Tumours of the Lung, Pleura, Thymus and Heart. Travis WD, Brambilla E, Müller-Hermelink HK and Harris CC (eds.) World Health Organization Classification of Tumours. IARC Press, Lyon, pp12-15, 2004.

2. Adjei AA: Blocking oncogenic ras signaling for cancer therapy. J Natl Cancer Inst 93: 1062-1074, 2001.

3. Yokota $\mathrm{J}$ and Kohno T: Molecular footprints of human lung cancer progression. Cancer Sci 95: 197-204, 2004.

4. Kiaris H and Spandidos DA: Mutations of ras genes in human tumours (review). Int J Oncol 7: 413-421, 1995.

5. Shigematsu H and Gazdar AF: Somatic mutations of epidermal growth factor receptor signaling pathway in lung cancers. Int J Cancer 118: 257-262, 2006.

6. Shibata T, Hanada S, Kokubu A, et al: Blackwell publishing Asia gene expression profiling of epidermal growth factor receptor/KRAS pathway activation in lung adenocarcinoma. Cancer Sci 98: 985-991, 2007.

7. Paez JG, Jaånne PA, Lee JC, et al: EGFR mutations in lung cancer: correlation with clinical response to gefitinib therapy. Science 304: 1497-1500, 2004.

8. Lynch TJ, Bell DW, Sordella R, et al: Activating mutations in the epidermal growth factor receptor underlying responsiveness of non-small-cell lung cancer to gefitinib. N Engl J Med 350: 2129-2139, 2004.

9. Takano T, Ohe Y, Tsuta K, et al: Epidermal growth factor receptor mutation detection using high-resolution melting analysis predicts outcomes in patients with advanced nonsmall cell lung cancer treated with gefitinib. Clin Cancer Res 13: 5385-5390, 2007.

10. Krypuy M, Newnham GM, Thomas DM, Conron M and Dobrovic A: High resolution melting analysis for the rapid and sensitive detection of mutations in clinical samples: KRAS codon 12 and 13 mutations in non-small cell lung cancer. BMC Cancer 6: 295, 2006
11. Do H, Krypuy M, Mitchell PL, Fox SB and Dobrovic A: High resolution melting analysis for rapid and sensitive EGFR and KRAS mutation detection in formalin fixed paraffin embedded biopsies. BMC Cancer 8: 142, 2008.

12. Massarelli E, Varella-Garcia M, Tang X, et al: KRAS mutation is an important predictor of resistance to therapy with epidermal growth factor receptor tyrosine kinase inhibitors in non-small cell lung cancer. Clin Cancer Res 13: 2890-2896, 2007.

13. Soung YH, Lee JW, Kim SY, et al: Mutational analysis of EGFR and K-RAS genes in lung adenocarcinomas. Virchows Arch 446: 483-488, 2005.

14. Shigematsu H, Lin L, Takahashi T, et al: Clinical and biological features associated with epidermal growth factor receptor gene mutations in lung cancers. J Natl Cancer Inst 97: 339-346, 2005.

15. Siegfried JM, Gillespie AT, Mera R, Casey TJ, Keohavong P, Testa JR and Hunt JD: Prognostic value of specific KRAS mutations in lung adenocarcinomas. Cancer Epidemiol Biomarkers Prev 6: 841-847, 1997.

16. Keohavong P, DeMichele MAA, Melacrinos AC, Landreneau RJ, Weyant RJ and Siegfried JM: Detection of K-ras mutations in lung carcinomas: relationship to prognosis. Clin Cancer Res 2: 411-418, 1996.

17. Rosell R, Monzó M, Pifarré A, et al: Molecular staging of nonsmall cell lung cancer according to K-ras genotypes. Clin Cancer Res 2: 1083-1086, 1996.

18. Rodenhuis S and Slebos RJC: Clinical significance of ras oncogene activation in human lung cancer. Cancer Res 52: S2665-S2669, 1992.

19. Sakuma Y, Matsukuma S, Yoshihara M, et al: Distinctive evaluation of non-mucinous and mucinous subtypes of bronchioloalveolar carcinomas in EGFR and K-ras gene-mutation analyses for Japanese lung adenocarcinomas. Am J Clin Pathol 128: 100-108, 2007.

20. Baea NC, Chaeb MH, Leec MH, et al: EGFR, ERBB2, and KRAS mutations in Korean non-small cell lung cancer patients. Cancer Genet Cytogenet 173: 107-113, 2007.

21. Wu C, Hsu H, Liu H, et al: Reversed mutation rates of KRAS and EGFR genes in adenocarcinoma of the lung in Taiwan and their implications. Cancer 113: 3199-3208, 2008.

22. Tam IY, Chung LP, Suen WS, et al: Distinct epidermal growth factor receptor and KRAS mutation patterns in non-small cell lung cancer patients with different tobacco exposure and clinicopathologic features. Clin Cancer Res 12: 1647-1653, 2006.

23. Gautschi O, Huegli B, Ziegler A, et al: Origin and prognostic value of circulating KRAS mutations in lung cancer patients. Cancer Lett 254: 265-273, 2007

24. Hunt JD, Strimas A, Martin JE, et al: Differences in KRAS mutation spectrum in lung cancer cases between AfricanAmericans and Caucasians after occupational or environmental exposure to known carcinogens. Cancer Epidemiol Biomarkers Prev 11: 1405-1412, 2002.

25. Zhou L, Wang L, Palais R, Pryor R and Wittwer CT: Highresolution DNA melting analysis for simultaneous mutation scanning and genotyping in solution. Clin Chem 51: 1770-1777, 2005.

26. Nomoto K, Tsuta K, Takano T, et al: Detection of EGFR mutations in archived cytologic specimens of non-small cell lung cancer using high-resolution melting analysis. Am J Clin Pathol 126: 608-615, 2006.

27. Leeneer KD, Coene I, Popper B, Paepe AD and Claes K: Rapid and sensitive detection of BRCA1/2 mutations in a diagnostic setting: comparison of two high-resolution melting platforms. Clin Chem 54: 982-989, 2008.

28. Koeffler HP, McCormick F and Denny C: Molecular mechanisms of cancer. West J Med 155: 505-514, 1991.

29. Der CJ, Krontiris TG and Cooper GM: Transforming genes of human, bladder and lung carcinoma cell lines are homologous to the ras genes of Harvey and Kirsten sarcoma viruses. Proc Natl Acad Sci USA 79: 3637-3640, 1982.

30. Wong CE, Karnezis AN, Rosario RD, Lauro RD and Balmain A: Kras regulatory elements and exon $4 \mathrm{~A}$ determine mutation specificity in lung cancer. Nat Genet 40: 1240-1244, 2008.

31. Hsieh RK, Lim KH, Kuo HT, Tzen CY and Huang MJ: Female sex and bronchioloalveolar pathologic subtype predict EGFR mutations in non-small cell lung cancer. Chest 128: 317-321, 2005.

32. Zhang G, Lin J, Wang Z, et al: Epidermal growth factor receptor double activating mutations involving both exons 19 and 21 exist in Chinese non-small cell lung cancer patients. Clin Oncol 19: 499-506, 2007. 
33. Mu X, Li L, Zhang X, et al: Gefitinib-sensitive mutations of the epidermal growth factor receptor tyrosine kinase domain in chinese patients with non-small cell lung cancer. Clin Cancer Res 11: 4289-4294, 2005.

34. Wang Z, Wu YL, Zhang GC, Zhou Q, Xu CR and Guo AL: EGFR KRAS mutations and gefitinib therapy in chinese NSCLC patients. Onkologie 31: 174-178, 2008.

35. Calvez FL, Mukeria A, Hunt JD, et al: TP53 and KRAS mutation load and types in lung cancers in relation to tobacco smoke: distinct patterns in never, former, and current smokers. Cancer Res 65: 5076-5083, 2005.
36. Riely GJ, Kris MG, Rosenbaum D, et al: Frequency and distinctive spectrum of KRAS mutations in never smokers with lung adenocarcinoma. Clin Cancer Res 14: 5731-5734, 2008.

37. Reed GH and Wittwer CT: Sensitivity and specificity of singlenucleotide polymorphism scanning by high-resolution melting analysis. Clin Chem 50: 1748-1754, 2004.

38. Liew M, Pryor R, Palais R, Meadows C, Erali M, Lyon E and Wittwer C: Genotyping of single-nucleotide polymorphisms by high-resolution melting of small amplicons. Clin Chem 50: 1156-1164, 2004. 